10.2478/gb-2021-0017

sciendo

Die Angst war „Ein Gefängnis ohne Mauern"1 Rezension zu: Spiridon-Șerbu, Claudia: Zensur in der rumäniendeutschen Literatur der 1970er und 1980er-Jahre.

Wien: LIT Verlag 2018. ISBN 978-3-643-90998-5.

Reihe: Osteuropa Bd. 12., 272 S.

\title{
Maria SASS
}

Prof. Dr., Lucian-Blaga-Universität Hermannstadt/Sibiu; E-Mail:maria.sass@ulbsibiu.ro

Abstract:The present article is an interpretation of Claudia Spiridon-Șerbu's study on censorship in Romania during the last 30 years of communist rule. Drawing on unreleased documents from the CNSAS (National Council for the Study of the Securitate Archives) Archive dwelling on German ethnic authors from Romania, the author paints a vivid picture of the complex phenomenon of literary censorship. The study follows both the official censorship undertaken by the General Office for Press and Publishing and the unofficial prosecution of writers by Securitate agents and their collaborators.

Keywords: communist censorship, prosecution file, CNSAS

Dieses Buch stellt die überarbeitete Fassung der Dissertation von Claudia Spiridon-Șerbu dar, die im September 2017 an der Babeș-Bolyai-Universität vorgelegt und verteidigt wurde. Wie schon im Titel angekündigt wird, handelt es sich um eine Untersuchung der kommunistischen Zensurpraxis in der rumäniendeutschen Literatur der 1970er und 1980er-Jahre.

${ }^{1}$ Neustädter, Carol, zit. nach: Spiridon-Șerbu, Claudia: Zensur in der rumäniendeutschen Literatur der 1970er und 1980er Jahre. Wien: LIT Verlag 2018. ISBN 978-3-643-90998-5. Reihe: Osteuropa Bd. 12., S. 133. 
In einem einführenden Kapitel werden die theoretischen Grundlagen zum Thema Zensur angeführt, die Autorin knüpft an Peter Dittmars ${ }^{2}$ Ansatz zum Zensurbegriff an, der folgend definiert wird: „Zensur besteht dann, wenn eine eingerichtete Behörde nach vorgegebenen Vorschriften, jeden Text vor seiner Veröffentlichung überprüftundüberdessen Druckgenehmigung, dessen Verbot oder Änderung entscheidet." Gleichzeitig erklärt die Autorin, dass ausschließlich der Zensurapparat des kommunistischen Systems und dessen Auswirkungen auf das Werk der Schriftsteller analysiert werden sollen.

Die Studie der Claudia Spiridon-Șerbu beruht auf Archivmaterialien, Interviews mit Zeitzeugen und unveröffentlichte Texte. Untersucht werden Quellen aus dem CNSAS-Archiv, deren Durchsicht seit Mitte der Jahre 2000 möglich war. Die Zensurpraktiken werden aus doppelter Perspektive erläutert: Einerseits wird das rumänische Zensursystem näher beleuchtet, andererseits wird der deutschsprachige Diskurs der Zeit in Betracht gezogen.

Das Kapitel Die Anfänge der kommunistischen Zensur beschreibt die kulturpolitischen Rahmenbedingungen der Literaturszene im Nachkriegsrumänien, hervorgehoben wird, dass die Zensur im Laufe von 45 Jahren einem ständigen Wandel ausgesetzt gewesen ist. Der Beginn des Zensursystems deckt sich mit dem Anfang der kommunistischen Diktatur in Rumänien und der Machtübernahme der Sowjetunion über den Osten Europas. Alle neugegründeten sozialistischen Staaten sollten nach russischem Muster den sozialistischen Realismus nachahmen, laut dem eine militante Literatur zur Propagierung von kommunistischen Werten gefordert wurde. Somit schlugen sich die Richtlinien des sozialistischen Realismus sowohl in der rumänischen als auch in der Literatur der Minderheiten nieder,

2 Dittmar, Peter: Lob der Zensur. Verwirrung der Begriffe. Verwirrung der Geister. Köln: Kölner Universitätsverlag 1982, zit. nach: SpiridonŞerbu, Claudia, S.20. 
obgleich auf unterschiedlicher Weise. Zum Unterschied von den rumänischen Schriftstellern waren die rumäniendeutschen Autoren, beginnend mit der Regierung von Gheorghe GheorghiuDej, mit einem problematischen Erbe konfrontiert, das durch die deutsche Sprache bedingt war: Diese hatten nationalsozialistische Überlieferungen aufzuarbeiten und die eigenen literarischen Produktionen nach den sozialistischen Vorschriften zu orientieren. Deutsche literarische Schriften wurden streng zensiert und zur Einschüchterung wurden viele Schriftsteller in Schauprozessen verurteilt.

In mehreren Unterkapiteln wird die sozial-politische und kulturelle Situation in Rumänien erläutert, einige Änderungen werden nach 1965 verzeichnet, nachdem Ceaușescu an die Macht kam, der eine scheinbare Öffnung zum Westen bekannt gab. Zwar ist die Zahl der Prozesse gegen Schriftsteller stark gesunken, doch jede Art von Opposition wurde unterdrückt und die in der Verfassung festgehaltene Meinungsfreiheit blieb weiter bloß Schein. Somit war die Einhaltung des sozialistischen Realismus verpflichtend, gestaltet werden sollte die sozialistische Wirklichkeit, wobei parteifeindliche, unmoralische Einstellungen oder angebliche Gefährdung der sozialistischen Gesellschaft durch Publikationsverbot bestraft wurden.

Die nächsten zwei Kapitel beziehen sich auf die Zensur in der Ceaușescu-Diktatur, von 1965 bis zum Fall des Eisernen Vorhangs 1989. In dieser Zeitspanne wurde der Begriff ,sozialistischer Realismus" vermieden, doch blieben als Basis der durch die kommunistische Partei akzeptierte künstlerische Produktion, Wirklichkeitsnähe und Volksverbundenheit, die Literatur musste sich nach einem Massenpublikum richten und den Diktator würdigen.

Claudia Spiridon-Șerbu beschreibt eingehend die Struktur und Funktionsmechanismen der offiziellen Zensurbehörde nach 1965 und zeigt, dass alle Werke, die gedruckt werden sollten, eine Druckgenehmigung brauchten, an der Zensur waren 
neben der offiziellen Behörde Verlagslektoren und Redakteure mitbeteiligt. Die Autorin dokumentiert die Art und Weise, wie die Zensur durchgeführt wurde, als Quelle verwendet sie das Archiv der Allgemeinen Abteilung für Presse und Druckerzeugnisse. Die Dokumentensammlung deckt dieFunktionsmechanismen der Behörde auf, enthüllt die Aufgaben der Zensoren und die Typologie ihrer Eingriffe und offenbart Texte der rumäniendeutschen Literatur, die zensiert wurden. Obwohl alles als sehr streng geregelt erscheint, betont Spiridon, dass das Zensurverfahren meistens erschwert war, weil genaue Zensurkriterien fehlten. Die Zensur reagierte beispielsweise auf Reizwörter (Unfreiheit, Beschränkung, Emigration, Zensur, Sex) oder auf verpönte Themen (Russlanddeportation).

Anhand von ausgewählten Beispielen -Verona Bratesch, Wolf von Aichelburg, Bernd Kolf, Gerhard Ortinau-illustriert die Autorin Eingriffe der Zensur und offenbart rumäniendeutsche literarische Texte, die zensiert wurden. Analysiert wird der Band Wiege im All (1971) der Kronstädter Lyrikerin und Übersetzerin Verona Bratesch (1922-1991), für den ein Gutachten „gegen die Veröffentlichung des Bandes ${ }^{\text {ee }}$ vorliegt. Weiter werden Gedichte, die von der Zensur aus einem Band Wolf von Aichelburgs (1912-1994) entfernt werden, analysiert. Bern Kolfs Bände Zwischen sieben und unendlich (1971) und die bewohnbarkeitdes Mondes (1976) erhielten Publikationsverbot. Die Gründe dafür waren „Ambiguität, Ironie, Kritik" (Spiridon, 97). Aus der Akte Gerhard Ortinaus ist zu entnehmen, dass der Band verteidigung des kugelblitzes. kurze prosa (1976) keine Druckgenehmigung erhielt.

Claudia Spiridon erklärt auch, dass trotz der schwierigen Situation der Zensur es Möglichkeiten gegeben hat, literarische Werke von hoher ästhetischer Qualität zu veröffentlichen. Diesbezüglich kam den Verlagslektoren eine bedeutende Rolle zu. Als Fallbeispiele werden Gerhard Ortinau, Rolf Bossert, Anemone Latzina, Franz Hodjak und Bernd Kolf analysiert. 
Aus dem CNSAS-Archiv nimmt die Autorin Dokumente, die sich auf den Dacia-Verlag beziehen, auf und erklärt, dass Franz Hodjak, Lektor des Dacia-Verlags in Klausenburg, „die Verbreitung einer kritisch orientierten und weltoffenen Literatur" (Spiridon: 107) unterstützte, in ihn erkannten Literaturwissenschaftler und Rezensenten der Zeit den „Förderer einer neuen Generation rumäniendeutscher Dichter.“ (Spiridon:107) Als Verlagslektor hatte Franz Hodjak hohe Maßstäbe im DaciaVerlag eingeführt, die zu beachten waren, er richtete sich gegen die konservative siebenbürgische Heimatliteratur und förderte das Eindringen von Einflüssen aus der modernen binnendeutschen Literatur.

In den meistens Fällen gab es eine enge Zusammenarbeit zwischen dem Autor, Redakteur und Verleger, man trachtete danach, zu einem Ergebnis zu gelangen, dass Bücher bei der Zensur durchkamen. Trotzdem gibt es Gutachten für Werke, die keine Druckgenehmigung erhielten, die Autorin bietet großen Raum der Analyse solcher Werke, die von der Zensur entweder abgelehnt oder verändert wurden.

Neben der Zensurbehörde kam dem Geheimdienst eine entscheidende Rolle im Zensurverfahren, die Angst sollte die Grundlage des Kontrollapparats sein. Mit der Übernahme der Macht durch Ceaușescu, wird die blutige Vorgehensweise des Stalinismus zugunsten einer psychologischen, prophylaktischen Unterdrückungsstrategie aufgegeben. Die Grundidee bestand darin, eine auf Angst und Einschüchterung basierende „präventive Politik“ (Spiridon: 134) zu führen. Es wurden keine Leute mehr im Gefängnis umgebracht, sondern es wurde dafür gesorgt, dass es keinen Grund mehr gab, Leute zu verhaften.

Bei der Untersuchung der Dokumentensammlung des CNSAS (Archiv des ,Nationalen Rates zum Studium der Unterlagen des kommunistischen Geheimdienstes") für die Zeitspanne 1970-1989 kommt zum Vorschein, dass fast alle Schriftsteller und Redakteure der rumäniendeutschen Literaturszene im Visier 
der Securitate gestanden haben. Diese Tatsache bestätigt nochmals die Komplexität des Kontrollsystems. Gründe dafür waren kritikbeladene Schriften, das Thema der Emigration, der Kontakt zu Personen aus der Bundesrepublik oder die Veröffentlichung von literarischen Werken im Westen.

Im vierten Kapitel analysiert Spiridon Quellen und Berichte der Securitate-Offiziere, um zu beleuchten, ob diese tatsächlich in dem Zensursystem impliziert waren oder ob sie nur eine vorbeugende Rolle gespielt haben. Doch hebt die Autorin hervor, dass der Sicherheitsdienst unabhängig vom Tätigkeitsbereich der „Allgemeinen Abteilung für Presse und Druckerzeugnisse“ funktionierte, auf Grund der überlieferten Akten geht hervor, dass es keine Zusammenarbeit zwischen der Securitate und der offiziellen Zensurbehörde gab. Neben den offiziellen Angestellten des Geheimdienstes spielten in der Kontrolle und dem Verbieten von Manuskripten eine bedeutende Rolle die inoffiziellen Mitarbeiter bzw. die Kollaborateure oder Informanten. Die Securitate beaufsichtigte alle Teilgebiete de Buchwesens, es wurden Verfolgungsakten, die auf die Schreibtätigkeit verschiedener Autoren fokussiert waren, eröffnet und eine komplexe Vorgehensweise der Securitate bekunden. Die Informanten der Securitate waren entweder Schriftsteller, Verlagslektoren, Redaktionsmitglieder von Zeitungen und Zeitschriften oder stammten aus den Freundeskreisen der Autoren. In den Berichten war die politische Beurteilung oft überspitzt, die Berichterstatter verfolgten ein einziges Ziel: einen guten Eindruck auf Menschen in führenden Positionen zu machen, zum Zweck des Aufsteigens in der Hierarchie oder aus Angst, nicht selbst verdächtigt zu werden. Claudia Spiridon bezieht sich in ihrer Analyse auf, von Kollaborateuren verfasste kompromittierende Berichte für Schriftsteller wie Johann Lippet, Gerhard Ortinau, Richard Wagner, Horst Samson, William Totok, Bern Kolf, Anemone Latzina, Nikolaus Bernwanger, Helmuth Frauendorfer. 
Die inoffiziellen Mitarbeiter wurden vom Geheimdienst aus Zeitungs- und Zeitschriftenredaktionen angeworben und hatten die Aufgabe, verdächtige Manuskripte bei der Securitate einzureichen, noch bevor diese in die Redaktion des Verlags oder der Zeitschrift eingereicht wurden. Die Führungsoffiziere erwarteten von ihren "Quellen“, „die potenzielle Sprengkraft eines noch nicht publizierten Textes [zu] erkennen und dessen Verbreitung [zu] unterbinden" (Spiridon: 144).

In der Arbeit werden einige Verfolgungsakten analysiert, mehrere Zeitschriftenredakteure waren zu Mitarbeitern der Securitate geworden. Ein Beispiel dafür ist ein Redakteur der Zeitschrift Echinox, mit dem Decknamen Walter, der regelmäBig Berichte bei der Securitate einreichte. Aufgrund solcher Berichte wurden beispielsweise Manuskripte von J. Lippet abgelehnt und bekamen keine Druckgenehmigung. Kompromittierende Berichte wurden ab 1972 auch über Richard Wagner, in dessen Fall Schubladenliteratur angemeldet wird, bei der Securitate eingereicht. Auch andere Mitglieder der Aktionsgruppe Banat befinden sich im Visier der Securitate. Über William Totok gemachten Denunziation führten zur Verhaftung des Dichters und zu acht Monaten Gefängnis; ständig bespitzelt und mit geringen Publikationsmöglichkeiten führten zum Entschluss, dass Gerhard Ortinau 1980 das Land endgültig verließ; Publikationsverbot erhielt auch Helmuth Frauendorfer.

In ihrer Analyse des kommunistischen Kontrollsystems weist Spiridon auf mehrere Verfahren des Sicherheitsdienstes hin. So z. B. war die Securitate darauf angewiesen, Informanten in den Redaktionen der Verlage und Zeitungen einzuschleusen, um die Verbreitung unerwünschter Texte zu blockieren. Bei der Rekrutierung und Einsetzung von inoffiziellen Mitarbeitern ging es darum, zu den Manuskripten zu kommen, bevor das Druckverfahren eingeleitet worden wäre. Doch ließen sich nicht alle Redaktionsmitglieder einschüchtern, es gab auch Redakteure, die sich auf der Seite der Autoren platzierten, beispielsweise 
Bernd Kolf, ehemaliger Redakteur der Karpatenrundschau. Demnach wurde er ab 1972 ständig beobachtet, und vor seiner Ausreise in die Bundesrepublik konnte er nur zwei Bände veröffentlichen (Zwischen sieben und unendlich (1971) und die bewohnbarkeit des Mondes: gedichte, aphorismen und notate (1976). In einer ähnlichen Situation befand sich Nikolaus Bernwanger, Chefredakteur der Neuen Banater Zeitung. Eine weitere Lyrikerin, die sich nicht scheute, ihre Kritik gegen das Regime zu richten und Stellung zu beziehen, war Anemone Latzina. Sie war Redakteurin der Neuen Literatur und bediente sich ihrer Position, ,um Gedichte herauszubringen, die gesellschaftlich-politische Zusammenhänge akkurat reproduzierten und die Scheinlegitimität des Kommunismus hinterfragten.“ (Spiridon: 197). Im Zensurprozess hatten die Redakteure und Verlagslektoren Schlüsselpositionen. Es hing von deren Bereitschaft und deren Mut ab, ob ein kritischer Text gedruckt wurde oder nicht. Anemone Latzina erwies sich diesbezüglich als eine Verbündete der Schriftsteller, doch konnte sie wegen der Verfolgung nur einen einzigen Band 1971 veröffentlichen. Erwähnt werden soll auch der bekannte Literaturwissenschaftler Gerhardt Csejka, der auch eine Verfolgungsakte hatte und als Redakteur der Neuen Literatur gegen die vorprogrammierte Parteilyrik und gegen die hölzerne Sprache kämpfte.

Ausgehend von Berichten konnte der Grund für Publikationsverbote ermittelt und festgestellt werden, welches die Maßnahmen waren, die gegen die Schriftsteller getroffen wurden. Konnten die Kollaborateure keine Druckvorlagen einreichen, wurden von der Securitate Hausdurchsuchungen organisiert und Manuskripte beschlagnahmt. Solche Hausdurchsuchungen erlebten in der untersuchten Zeitspanne William Totok, Horst Samson, Rolf Bossert. Die Dokumentensammlung der ehemaligen Securitate-Akten deckt zahlreiche Texte auf, Lyrik und Prosafragmente zugleich, die in den 1970er und 1980er-Jahre unterbunden wurden. 
Zusammenfassend lässt sich sagen, dass die Securitate-Offiziere in den Besitz der noch nicht veröffentlichten Manuskripte gelangten, noch bevor diese zu der Zensurbehörde ankamen. Die Zusammenarbeit der Securitate mit den Redakteuren der Zeitschriften Neue Literatur, Neue Banater Zeitung, Karpatenrundschau oder Echinox führte zur Verhinderung von zahlreichen Texten, die den Vorschriften der Parteilyrik nicht entsprachen.

$\mathrm{Zu}$ den von der Geheimpolizei eingesetzten Maßnahmen gehörten: Mahnungen, Verhöre, Einschüchterungen, Publikationsverbot, Exmatrikulation oder Verlust des Arbeitsplatzes. Die Macht der Securitate wirkte einerseits mittels ihrer inoffiziellen Mitarbeiter direkt auf die Literaturszene ein, andererseits agierte der Securitate-Terror indirekt auf das Gedächtnis der Autoren und Verlagslektoren und blockierte vorbeugend jede Kritik an dem Regime. Dieses permanente Schikanieren führte zur Selbstzensur, die von Joachim Walther folgendermaßen zusammengefasst wurde: ,gesellschaftlicher Angst-Raum [...] der ohne direkte Repression, unsichtbar, subtil, als Selbstzensur, als Denkblockade und als schwarzer Fleck."3(Spiridon: 224) Die Mitarbeiter der Securitate durften nicht selbst Textänderungen unternehmen, doch misshandelten sie psychologisch die Autoren. Indem sich die formelle Zensur auf die Überprüfung der Werke von einer eingerichteten Instanz beschränkte, berief sich die informelle Zensur auf die Ausübung der Gewalt. Die Selbstzensur funktionierte auf der Ebene des Unbewussten, Gedanken wurden nicht rational, sondern instinktiv zensiert, was eindeutig das Schaffensvermögen einschränkte. Der Autor übersah nach der Niederschrift sein Werk nicht, um Textstellen auszustreichen, dieser Prozess war tief im Gedächtnis eingebettet. Folglich wurden künstlerische Einfälle unterdrückt und vernichtet.

3 Walther, Joachim: Der fünfte Zensor. Unterdrückte Literatur in der DDR. In: Zensur. Hg. von Rosenthal, Claudius. Konrad-AdenauerStiftung, Sankt-Augustin 2003, S.77-101, hier.zit. S.87. 
Selbstzensur ist dadurch zu erklären, dass die Autoren mithilfe der Selbsteinschränkungen der institutionalisierten Zensur entgehen wollten, um sich die Veröffentlichungsmöglichkeiten zu verbessern.

Obwohl das Zensuramt nach 1977 aufgelöst wurde, gab es eine „Verantwortung der Angst“" (Spiridon: 227), die Angst vor Abmahnungen oder vor Publikationsverbot bildete sich zu einer inneren Selbstkontrolle aus. Die Zensurfälle, zu denen man heute Zugang in Archiven hat, zeigen die Wechselwirkungen von Kontrolle, Kompromiss und Kooperation, die die Beziehungen zwischen Autoren, Verlagslektoren und Zensoren geprägt haben. Dennoch gab es Möglichkeiten, im Kontext der Zensur Texte zu publizieren, die sich den politischen Richtlinien entzogen.

Die Arbeit von Claudia Spiridon-Șerbu vermittelt ein Bild des komplexen Zensurphänomens in Rumänien und bringt unterschiedliche Aspekte ein, die bis jetzt ungenügend erforscht wurden. Es geht nicht um die Dekonstruktion von Feindbildern, sondern um das Schaffen eines Blicks auf die Diktatur. Schlussfolgernd betont die Autorin, ihre Arbeit sei als Beitrag zur Aufarbeitung der Vergangenheit und zur Vergangenheitsbewältigung zu verstehen. 